\title{
Immunological evidence for increased oxidative stress in diabetic rats
}

\author{
N. Traverso ${ }^{1}$, S. Menini ${ }^{1}$, L. Cosso ${ }^{1}$, P. Odetti ${ }^{2}$, E. Albano ${ }^{3}$, M. A.Pronzato ${ }^{1}$, U. M. Marinari ${ }^{1}$ \\ ${ }^{1}$ Institute of General Pathology, University of Genova, Italy \\ ${ }^{2}$ Department of Internal Medicine, University of Genova, Italy \\ ${ }^{3}$ Department of Medical Sciences-Novara, University of Turin, Italy
}

\begin{abstract}
Summary The role of oxidative stress in aging and diabetes mellitus is currently under discussion. We previously showed age-dependent accumulations of fluorescent protein adducts with lipoperoxidative aldehydes, (malondialdehyde (MDA), and hydroxynonenal (HNE)) in rat skin collagen with diabetic BB rats exhibiting faster accumulation. Modified proteins have been shown to be immunogenic: antibody titres against rat serum albumin modified by MDA and HNE (MDA-RSA and HNE-RSA) or oxidized by reactive oxygen species were measured by ELISA as markers of oxidative damage in BB diabetic and non-diabetic rats. Each tested antibody titre was significantly higher in the diabetic than in the non-diabetic rats. A significant correlation existed between anti-MDA-RSA and anti-HNE-RSA antibody titers. Only the anti-HNE-RSA antibody titre increased significantly with age $(p=0.052)$ in diabetic animals, while no titres increased significantly in non-diabetic animals. A major factor which correlated with the de-
\end{abstract}

velopment of these antibodies was diabetes duration: this was significant $(p=0.032)$ for anti-HNE-RSA antibody titre and slightly significant $(p=0.05)$ for anti-MDA-RSA antibody titre. Thus, chronic hyperglycaemia is probably fundamental in the increase of oxidative stress. There is correlation between anti-aldehyde-RSA antibody titres and the corresponding aldehyde-related collagen-linked fluorescence: modified collagen may play a part in the observed immune response. Our data indicate a stronger immune response of diabetic rats against proteins modified by lipoperoxidative aldehydes and oxygen free radicals, and they support the hypothesis of increased oxidative damage in diabetes. [Diabetologia (1998) 41: 265-270]

Keywords Oxidation, malondialdehyde, hydroxynonenal, gamma-irradiation, autoantibody, fluorescence, modified protein.
Oxidative stress [1] and non-enzymatic glycation [2] are thought to play fundamental roles in the pathogenesis of aging and the sequelae of diabetes mellitus, particularly through progressive damage to proteins. Oxidation can damage proteins either directly [3] or indirectly, through lipoperoxidative production of re-

Received: 6 August 1997 and in revised form: 15 October 1997

Corresponding author: Dr. N. Traverso, Institute of General Pathology, Via L. B. Alberti 2, I-16132 Genova, Italy Abbreviations: MDA, Malondialdehyde; HNE, 4-hydroxynonenal; ROS, reactive oxygen species; RSA, rat serum albumin; MDA-RSA, RSA modified by MDA; HNE-RSA, RSA modified by HNE; ROS-RSA, RSA modified by ROS. active aldehydes (e.g. malondialdehyde (MDA), and 4-hydroxy-2-nonenal (HNE), which react with proteins to generate fluorescent adducts [4]; glycation also leads to formation of fluorescent end-products [5]. Fluorescence measurements showed a gradual accumulation of glycation - and oxidation - end products in rat collagen during aging [6]; moreover, an acceleration of this process was detected in spontaneously diabetic $\mathrm{BB}$ rats [7]. While a large amount of data exist on specific glycation end products, and several molecular structures are already well known [8], there is less information on the role of oxidation in protein modification in vivo, due to the scanty knowledge about the structures of oxidative end products. 
Protein non-enzymatic modifications are able to generate immunogenic epitopes [9]; more recently the immunogenicity of proteins modified by MDA, HNE or oxidation has been proved [10]. It is conceivable that the appearance of non-enzymatically modified proteins in living organisms could elicit an immunological response, the intensity depending on the entity of the stimulation [11]. Atherosclerotic [11], hypertensive [12], uraemic [13] and non-insulindependent diabetic patients [14] showed higher antibody titres against oxidized LDL and MDA modified LDL than control subjects indicating an increased oxidative stress in these pathologies.

This study deals with the presence and the titres of different antibodies against oxidatively modified proteins in spontaneously diabetic rats. In particular, the antibody titre against MDA- and HNE-protein adducts and against proteins directly modified by reactive oxygen species (ROS) were evaluated. The detection of these antibodies is indirect proof of the occurrence of oxidatively modified proteins in vivo.

\section{Materials and methods}

Animals. We purchased 25 diabetes prone BB/Wor/Mol/BB male rats from Möllegaard Ltd (Skensved, Denmark) which were randomly caged in groups of 2 and fed with a standard diet (Piccioni Spa, Milan, Italy). We used 12 diabetes resistant $\mathrm{BB} / \mathrm{Wor} / \mathrm{Mol} / \mathrm{WB}$ rats as a control group.

The animals were maintained at $22-24^{\circ} \mathrm{C}$ with a $12 \mathrm{~h}$ dark/ light cycle, following common procedures for good animal care. Blood glucose was determined with reactive strips (Haemoglucotest; Boehringer Mannheim Italia Spa, Milan, Italy) and read with a reflectometer (Reflolux S; Boehringer Mannheim, Germany) 3 times a week by drawing blood from a tail vein.

A blood glucose level over $11 \mathrm{mmol} / \mathrm{l}$ was considered indicative of diabetes onset, which occurred at 8-12 weeks of age. Once diabetes was confirmed insulin therapy was begun, by means of subcutaneous implants of entrapped stearate insulin (slow release, 2 U/day for 30 days, Limplant; Linshin Inc., Scarborough, Canada) [7].

After a set duration of diabetes, the animals were anaesthetized $(65 \mathrm{mg} / \mathrm{kg}$ body weight sodium pentobarbital, intraperitoneal); blood was collected by heart puncture and aliquots were used for the final measurements of glycaemia; sera were allowed to separate and were stored at $-80^{\circ} \mathrm{C}$ until antibody tests were performed. The animals were killed while under anaesthesia and collagen-linked fluorescence was evaluated as previously described [6]. Briefly, a sample of subcutaneous connective tissue was removed, homogenized in phosphate buffered saline (PBS) $0.1 \mathrm{~mol} / 1$ ( $\mathrm{pH} 7.4)$, defatted by mild shaking overnight in chloroform/methanol (2:1) and washed in methanol and water. The remaining material was digested for $24 \mathrm{~h}$ at $37^{\circ} \mathrm{C}$ with mild shaking by purified collagenase (type VII; Sigma, St. Louis, Mo., USA) in HEPES buffer $(0.02 \mathrm{~mol} / \mathrm{l}, \mathrm{pH} 7.5)$ containing $0.01 \mathrm{~mol} / \mathrm{l} \mathrm{CaCl}_{2}$. The supernatant was used for the determination of hydroxyproline content [15] and for the registration of fluorescence at the wavelengths specific for MDA-protein adducts (390 nm excitation/460 nm emission) and for HNE-protein adducts (356 nm excitation/ $460 \mathrm{~nm}$ emission) [16].
Antigen preparation. Rat serum albumin (RSA, Sigma) $1.5 \mathrm{mg} /$ $\mathrm{ml}$ was incubated in PBS $10 \mathrm{mmol} / \mathrm{pH} 7.4$ with $20 \mathrm{mmol} / \mathrm{l}$ MDA sodium salt, prepared from tetraethoxypropane (Sig$\mathrm{ma}$ ), or with $5 \mathrm{mmol} / \mathrm{l} \mathrm{HNE}$, prepared from HNE diethylacetal kindly provided by Professor H. Esterbauer (Verkauf GmbH, München, Germany), or without any aldehydes, for $6 \mathrm{~h}$ at $37^{\circ} \mathrm{C}$ in the dark [17]. Unbound aldehydes were removed by rapid filtration through disposable desalting columns (molecular cut off $5 \mathrm{kDa}$; Pharmacia, Uppsala, Sweden); under these conditions a large fraction of epsilon amino groups of lysine residues were derivatized. The presence of MDA- and HNE-adducts in modified proteins was detected by fluorescence spectroscopy, using a Perkin-Elmer LS-5 spectrofluorometer: MDA-protein and HNE-protein adducts possess specific fluorescence at $390 \mathrm{~nm}$ excitation/460 nm emission and $356 \mathrm{~nm}$ excitation/460 $\mathrm{nm}$ emission respectively [16].

ROS modified RSA was obtained by gamma-irradiation $(10 \mathrm{krad})$ of a $1 \mathrm{mg} / \mathrm{ml}$ aqueous solution in a gamma-source (Pad.5; San Martino Hospital, Genova, Italy). Gamma-irradiation allows control of both the type and the concentration of radical species generated in the experimental samples [18]. Radiolytic yields are expressed as $\mathrm{G}$ values (micromolarity of $\mathrm{radical} / \mathrm{krad}$ ) [19]. In the radiolysis of water, the main species formed are $\mathrm{HO}^{\bullet}$ and $\mathrm{H}^{\bullet}$; the latter representing less than $10 \%$ of the generated radicals $\left(\mathrm{G}_{\mathrm{H}^{*}}=0.55\right)$, while hydroxyl radicals and solvated electrons are produced in similar amounts $\left(\mathrm{G}_{\mathrm{HO}}=\mathrm{G}_{\mathrm{e}-\mathrm{aq}}=3.0\right)$ [3]. In our experiments, gamma-irradiation was performed under air, in order to expose the proteins to similar concentrations of $\mathrm{HO}^{\bullet}$ and $\mathrm{O}_{2}{ }^{\bullet}(\mathrm{G}=3$ for both radicals) [20]; the doses of radiation were checked by Fricke dosimetry [21].

Measure of autoantibody titres. The evaluation of the different autoantibody titres was performed by ELISA. Disposable 96well polystyrene plates (Costar Italia srl, Milan, Italy) were used. Antigens for this assay included underivatized RSA, MDA-modified RSA (MDA-RSA), HNE-modified RSA (HNE-RSA) and ROS-modified RSA (ROS-RSA). Each well was coated with $10 \mu \mathrm{g}$ antigen in PBS $(0.1 \mathrm{~mol} / 1, \mathrm{pH} 7.4)$ for $4 \mathrm{~h}$ in the dark. The remaining binding sites were then blocked using $3 \%$ fetal bovine serum in PBS, for $2 \mathrm{~h}$ at $37^{\circ} \mathrm{C}$. In the present study a 1:20 dilution of serum from each rat was prepared and $200 \mu \mathrm{l}$ was added in duplicate to the wells coated with native and modified protein. After incubation at $37^{\circ} \mathrm{C}$ for $2 \mathrm{~h}$, the wells were decanted and washed 4 times with $0.25 \%$ Triton X-100 in PBS before a peroxidase-conjugated antibody (Sigma) specific for rat $\mathrm{IgG}$ (diluted 1:2000) was added. After $1 \mathrm{~h}$ incubation at $37^{\circ} \mathrm{C}$ and extensive washing, the peroxidase activity was developed using phenylendiamine dihydrochloride and $\mathrm{H}_{2} \mathrm{O}_{2}$ as revealing agents. The absorbance was measured at $492 \mathrm{~nm}$ in an automatic microplate reader. To calculate antibody titres, the ratio between the spectrophotometric readings of anti-modified and anti-native antigen wells was used: this approach minimizes the possible detection of false positive values due to cross-reactivity with both epitopes [14].

Statistical analysis. Statistical calculations included determination of mean \pm SEM, Student's $t$-test and evaluation of linear regression. Welch's correction, which does not assume equal variances, was applied when necessary. 


\section{Results}

The incubation of RSA with MDA or HNE gave rise to the production of intense fluorescence in the areas considered specific for the respective aldehyde adducts; this confirmed the derivatization of lysine residues with the two lipoperoxidative aldehydes. Characteristics of gamma-irradiated bovine serum albumin can be found in our previous paper [22]; RSA showed very similar fluorescence modifications after gamma-irradiation. No development of fluorescence was detected in RSA incubated in the absence of aldehydes (Table 1).

Evaluation of antibody titres against MDA-, HNE- and ROS-modified RSA lead to the observation that the distribution range of values was wider for diabetic BB rats than for control rats; the difference between diabetic and control rats was significant for each antibody titre evaluated. The highest significance was found for anti-MDA-RSA antibody titre (Fig. 1).

The statistical correlations between the various antibody titres were evaluated: a highly significant correlation was found between anti-MDA- and antiHNE-RSA antibody titres ( $p=0.0052$, Fig. 2$)$, and a significant correlation between anti HNE- and anti ROS-RSA antibody titres $(p=0.0101)$; however, no correlation was found between anti MDA- and anti ROS-RSA antibody titres.

Antibody titres were then analysed compared to the animal's age. Anti HNE-RSA antibody titre did not increase with age for non-diabetic animals $(n=9, r=0.184, p=0.64)$, while a significant increase was found for diabetic rats $(n=23, r=0.422$, $p=0.045$ ). Anti-MDA-RSA antibody titre did not increase with age in control animals, while an increase, although not significant, was found for diabetic rats. A slow, non significant increase of anti-ROS-RSA an-

Fig.1a-c. Anti MDA-RSA (a), anti HNE-RSA (b) and antiROS-RSA (c) antibody titres in control and diabetic rats. Antibody titres are evaluated by ELISA and calculated as the ratio between the spectrophotometric readings of anti-modified and anti-native antigen wells. Bars represent mean \pm SEM; ${ }^{*} p=0.0013 ;{ }^{* *} p=0.034 ;{ }^{* * *} p=0.022 ; n_{\text {control }}=9, n_{\text {diabetes }}=23$
Table 1. Development of fluorescence at the specific wavelengths in rat serum albumin after incubation with MDA $20 \mathrm{mmol} / \mathrm{l}$, HNE $5 \mathrm{mmol} / \mathrm{l}$, or without any aldehyde for $6 \mathrm{~h}$ at $37^{\circ} \mathrm{C}$

AUF (390 nm excitation/460 nm emission) $\begin{array}{ll}\text { without MDA } & \text { with MDA } \\ 10.1 \pm 2.1 & 760 \pm 12\end{array}$

AUF ( $356 \mathrm{~nm}$ excitation/460 $\mathrm{nm}$ emission) without HNE with $\mathrm{HNE}$ $5.2 \pm 1.7 \quad 90 \pm 7.5$

Data are mean \pm SEM

tibody titre with age was noticed for both non-diabetic and diabetic rats.

Antibody titre values were also analysed in relation to diabetes duration. Anti-HNE-RSA antibody titre increased significantly with diabetes duration; significance was higher than that of the relationship with animal age ( $p=0.032$, Fig. 3$)$. Analogously, antiMDA-RSA antibody titre showed an increase with diabetes duration, reaching slight significance $(p=0.05)$; if rats were divided into two groups based on diabetes duration (less and more than 5 months), a significant difference resulted $(p=0.017)$. No increase was found for anti-ROS-RSA antibody titre with diabetes duration.

Antibody titres against lipoperoxidative protein adducts were analysed compared to the corresponding collagen-linked fluorescence values obtained by subcutaneous connective tissue [7]. A high correlation coefficient resulted for the correlation between anti-MDA-RSA antibody titre and fluorescence at $390 \mathrm{~nm}$ excitation/460 $\mathrm{nm}$ emission, considered specific for MDA-protein adducts. Less strong, but still significant was the correlation between anti HNE-RSA antibody titre and fluorescence at $356 \mathrm{~nm}$ excitation/ $460 \mathrm{~nm}$ emission, considered specific for HNE-protein adducts (Fig. 4).

Gamma-irradiated albumin showed a maximum fluorescence development at $330 \mathrm{~nm}$ excitation/ $420 \mathrm{~nm}$ emission [22]; moreover a fluorescence peak was revealed in the subcutaneous collagen of diabetic rats [7] at the same wavelengths. Therefore, antiROS-RSA antibody titres were analysed compared to collagen-linked fluorescence at $330 \mathrm{~nm}$ excitation/
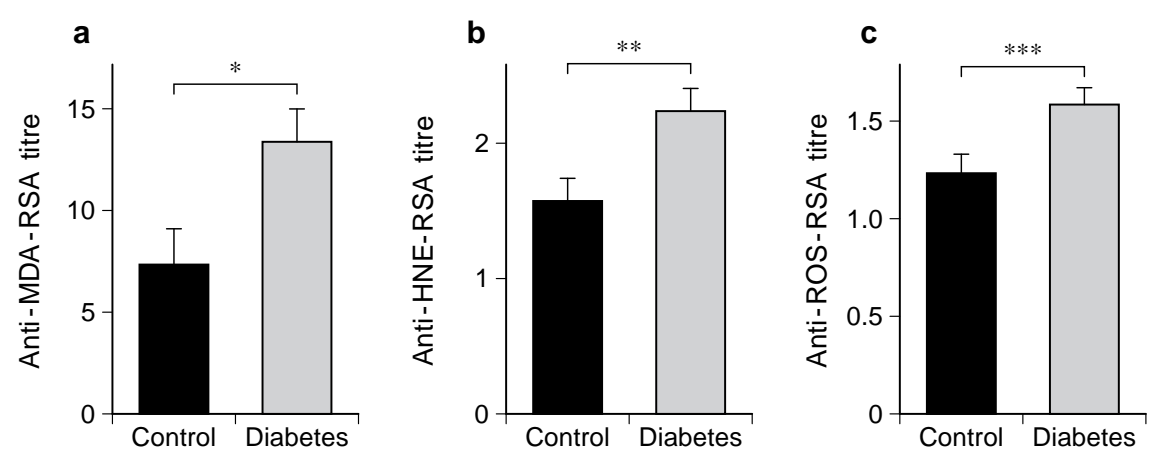


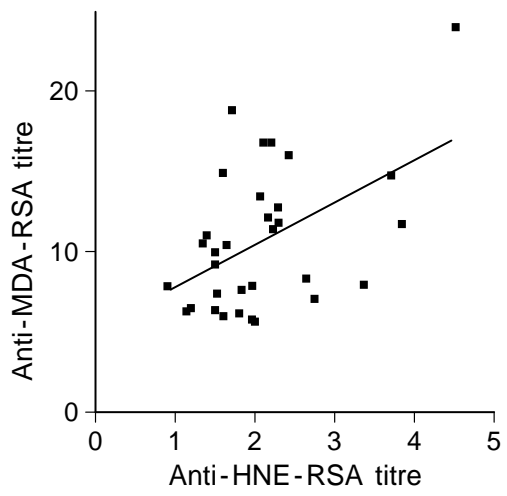

Fig. 2. Correlation between anti-HNE-RSA antibody titre and diabetes duration. The line indicates the better linear regression; $n=23, r=0.449, p=0.032$

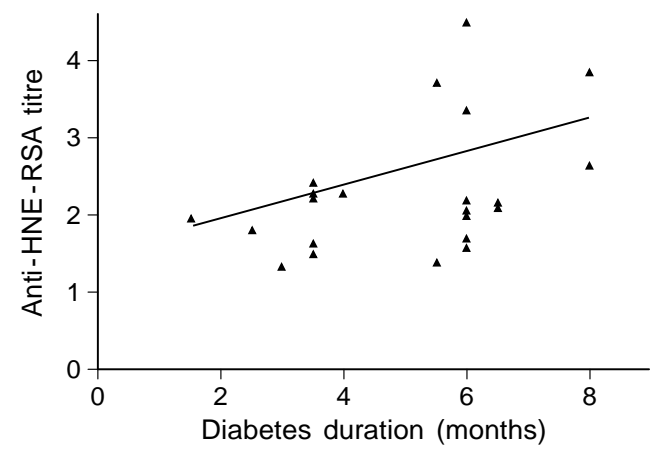

Fig. 3. Statistical correlation between anti-MDA-RSA and anti-HNE-RSA antibody titres. The line indicates the better linear regression; $n=32, r=0.482, p=0.0052$

$420 \mathrm{~nm}$ emission, but no correlation was found. Even semiquantitative analysis did not give any significant difference.

\section{Discussion}

While the role of non-enzymatic glycation modification of proteins in the development of diabetic sequelae is well documented [8], the involvement of oxidative stress has been suggested by many authors but doubts remain about its entity, causes and consequences. Glucose autoxidation [23] or glycoxidation [24] reactions can actually occur and could be responsible for increased oxidative stress in diabetic individuals, indicating a complex interaction between glycation and oxidation. Other mechanisms could also be involved, including impairment of energetic metabolism (particularly lipid metabolism), sorbitol pathways, inflammatory mediator production, antioxidants status [25], and availability of free metal ions [26].

In order to find evidence of increased oxidative stress in diabetes, various parameters, such as plasma MDA [27], $\mathrm{H}_{2} \mathrm{O}_{2}$ [28], and hydroperoxides [29], were
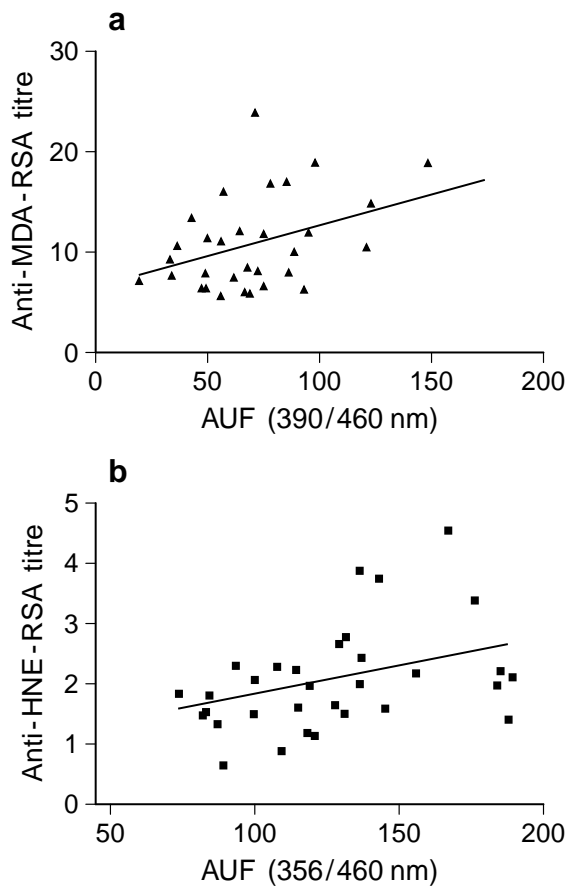

Fig. 4. a) Correlation between anti-MDA-RSA antibody titre and collagen-linked fluorescence related to MDA-adducts (390 $\mathrm{nm}$ excitation/460 $\mathrm{nm}$ emission). The line represents the better linear regression; $n=32, r=0.429, p=0.0143$. $b$ ) Correlation between anti-HNE-RSA antibody titre and collagenlinked fluorescence related to HNE-adducts (356 nm excitation/460 $\mathrm{nm}$ emission). The line represents the better linear regression; $n=32, r=0.371, p=0.04$

evaluated in diabetic humans or rats and were found to be significantly higher than in controls.

If oxidative stress is indeed increased in diabetic individuals, it is likely to damage protein per se, leading to "oxidation end products" which have not been well-characterized up to now. We showed an increase of fluorescence related to lipoperoxidative adducts in rat collagen during aging [6] and an acceleration of this phenomenon in diabetic rats [7]: this is a clue to indicate increased oxidative stress in diabetes. A more precise analysis would involve the evaluation of the levels of lipoperoxidative adducts, but unfortunately little information exists about these structures $[30,31]$. The immunologic approach is able to give indirect information about the existence of modified proteins, since they are immunogenic $[9,10]$. Each protein has its own half-life which is shorter in modified proteins, in particular oxidized proteins [32], even if data exist showing that multistep modifications can lead to proteins less susceptible to proteolysis [33]. It is conceivable that modified proteins elicit a significant immune response when they reach a threshold value; higher intensities of immune response should indicate higher levels of modified proteins [14].

It is noteworthy that almost all rats possess antimodified protein autoantibody titres higher than 1 in- 
dicating that oxidative modifications of proteins occur in vivo at such a rate as to elicit an immune response both in diabetic and non-diabetic animals.

The essential point of our results is that diabetic rats had significantly higher autoantibody levels against MDA-, HNE- and ROS-modified albumin. This should indicate an increased oxidative stress in diabetic rats, leading to increased generation of oxygen free radicals and lipoperoxidative aldehydes. Moreover, to our knowledge, there is no analysis of anti-HNE- or ROS-modified protein antibodies in diabetic organisms in the literature so far. The wide variation of autoantibody titres in diabetic rats could not be attributed to diversity in glycaemic equilibrium, since the rats were maintained as far as possible at the same glycaemic level, received the same type of insulin treatment and showed similar levels of glycated haemoglobin. In any case, the pattern of modified proteins in vivo is likely to be a mosaic due to the spectrum of non-enzymatic reactions on various proteins [34, 35]; the various epitopes generated on the proteins probably possess different antigenicity levels, leading to different immune responses.

A significant correlation with animal age was found only for anti-HNE-RSA antibodies in diabetic animals; non-diabetic rats did not show any significant increase in autoantibody titre with age; autoantibody levels, in general, could be expected to increase in old age, but no rats in this study were more than 12 months old. Diabetes duration turned out to be significantly correlated with both the autoantibodies against the aldehyde epitopes: this seems to underline the fundamental role of diabetes in the development of lipoperoxidation-modified proteins; therefore diabetes duration is more important than simple aging. Chronic hyperglycaemia is likely to be fundamental in the induction of increased oxidative stress, an accelerated consumption of antioxidants and probably an alteration in protein turnover capable of enhancing the accumulation of oxidatively modified proteins.

As far as the correlation of autoantibody titres with collagen-linked fluorescence is concerned, anti MDA-RSA antibody titre was highly significant, and a less intense, but still significant correlation was present for anti-HNE-RSA antibody titre. Collagenlinked fluorescence is likely to reflect several structural and conformational alterations due to various processes, including glycation, oxidation, formation of aldehyde adducts and so on. The fluorescence wavelengths typical for advanced glycation end products $(370 \mathrm{~nm}$ excitation/440 $\mathrm{nm}$ emission) are quite similar to the wavelengths typical for the aldehyde adducts. Though the existence of reciprocal interference between differently generated fluorescence peaks cannot be totally excluded, many data in the literature state the specificity of such fluorescence wavelengths $[6,38]$ which are considered indicative of the presence of the corresponding structures. The correlation of autoantibody titres with collagenlinked fluorescence indicates a parallel accumulation of modified collagen in tissues and of anti-modifiedRSA antibodies in plasma, but do not demonstrate that the antibodies have been elicited by modified collagen. However, these correlations suggest that collagen, after undergoing oxidative modification, could participate in the elicitation of the observed immune response. In this connection, it is interesting to remember that anti-MDA- or HNE-LDL antibodies, produced in guinea pigs, react with various other proteins, provided that they are modified in an analogous way so the epitopes appear to be constituted essentially by the aldehydes [10]; moreover, non-insulindependent diabetic patients, compared with healthy control subjects, showed higher anti-oxLDL and anti-MDA-LDL antibody titres, probably elicited by the presence of modified LDL and by chance crossreacting with modified HSA [14].

As for anti-ROS-RSA antibody titre, no correlation with collagen-linked fluorescence was found; this could depend on the fact that ROS-modified proteins could have particularly short half-lives, so that they may not be able to accumulate in parallel with antibody titre; moreover, the $330 \mathrm{~nm}$ excitation/ $420 \mathrm{~nm}$ emission fluorescence probably reflects more complex collagen modification than simple oxidation by ROS.

In order to shed further light on these issues, work is now in progress to evaluate the presence of antimodified collagen autoantibodies in diabetic rats and to distinguish IgG from IgM autoantibodies. Longitudinal studies are also under way to obtain information on the time course of the immune response against oxidatively modified proteins.

Acknowledgements. This work has been supported by grants from Progetto Strategico CNR n ${ }^{\circ}$ 96.04995.ST74 and MURST $30 \%$ and $60 \%$.

\section{References}

1. Harman D (1992) Free radical theory of aging. Mutat Res 275: 257-266

2. Monnier VM (1990) Nonenzymatic glycosylation, the Maillard reaction and the aging process. J Gerontol 45: B105-B111

3. Davies KJA (1987) Protein damage and degradation by oxygen radicals. I. General aspects. J Biol Chem 262 (20): 9895-9901

4. Esterbauer H, Cheeseman KH, Dianzani MU, Poli G, Slater TF (1982) Separation and characterization of the aldehydic products of lipid peroxidation stimulated by ADP$\mathrm{Fe}^{2+}$ in rat liver microsomes. Biochem J 208: 129-140

5. Bucala R, Cerami A, Vlassara H (1995) Advanced glycosylation end products in diabetic complications. Diabetes Reviews 3: 258-268

6. Odetti P, Pronzato MA, Noberasco G et al. (1994) Relationships between glycation and oxidation related fluorescences in rat collagen during aging. Lab Invest 70 (1): 61-67 
7. Odetti P, Traverso N, Cosso L, Noberasco G, Pronzato MA, Marinari UM (1996) Good glycemic control reduces oxidation and glycation end-products in collagen of diabetic rats. Diabetologia 39: 1440-1447

8. Monnier VM, Sell DR, Nagaraj RH et al. (1992) Maillard reaction-mediated molecular damage to extracellular matrix and other tissue proteins in diabetes, aging and uremia. Diabetes 41 [Suppl 2]: 36-41

9. Steinbrecher UP, Fisher M, Witzum JL, Curtiss LK (1984) Immunogenicity of homologous low density lipoprotein after methylation, ethylation, acetylation or carbamylation: generation of antibodies specific for derivatized lysine. J Lipid Res 25: 1109-1116

10. Palinski W, Ylä-Herttuala S, Rosenfeld ME et al. (1990) Antisera and monoclonal antibodies specific for epitopes generated during oxidative modification of low density lipoprotein. Arteriosclerosis 10: 325-335

11. Salonen JT, Ylä-Herttuala S, Yamamoto R et al. (1992) Autoantibodies against oxidized LDL and progression of carotid atherosclerosis. Lancet 339: 883-887

12. Maggi E, Marchesi E, Ravetta V, Martignoni A, Finardi G, Bellomo G (1995) Presence of autoantibodies against oxidatively modified low density lipoprotein in essential hypertension: a biochemical signature of an enhanced in vivo low density lipoprotein oxidation. J Hypertens 13 (1): 129_ 138

13. Maggi E, Bellazzi R, Gazo A, Seccia M, Bellomo G (1994) Autoantibodies against oxidatively-modified LDL in uremic patients undergoing dialysis. Kidney Int 46: 869-876

14. Bellomo G, Maggi E, Poli M, Agosta FA, Bollati P, Finardi G (1995) Autoantibodies against oxidatively modified low density lipoproteins in NIDDM. Diabetes 44: 60-66

15. Stegemann H, Stalder K (1967) Determination of hydroxyproline. Clin Chim Acta 18: 267-273

16. Carini R, Mazzanti R, Biasi F et al. (1988) Fluorescent aldehyde-protein adducts in the blood serum of healthy alcoholics. Adv Biosci 71: 61-64

17. Chen Q, Esterbauer H, Jürgens G (1992) Studies on epitopes on low density lipoprotein modified by 4-hydroxynonenal. Biochem J 288: 249-254

18. Asmus KD (1984) Pulse radiolysis methodology. Meth Enzymol 105: 167-178

19. Seccia M, Brossa O, Gravela E, Slater TF, Cheeseman KH (1991) Exposure of $\beta \mathrm{L}$-crystallin to oxidizing free radicals and Maillard reactions. Biochem J 274: 869-873

20. Gebicki S, Gebicki JM (1993) Formation of peroxide in amino acids and proteins exposed to oxygen free radicals. Biochem J 289: 743-749

21. Fricke H, Hart EJ (1966) Radiation dosimetry. In: Attix FH, Roesch WC (eds) Academic Press, New York, pp 167-170

22. Traverso N, Odetti P, Cheeseman K, Cottalasso D, Marinari UM, Pronzato MA (1996) Susceptibility of gamma-irradiated proteins to in vitro glycation: exposure to oxygen free radicals increases glycation-induced modifications. Cell Biochem Funct 14: 149-154

23. Wolff SP, Dean RT (1987) Glucose autoxidation and protein modification. Biochem J 245: 243-250

24. Wells Knecht MC, Thorps RS, Baynes JW (1995) Pathways of formation of glycoxidation products during glycation of collagen. Biochemistry 34 (46): 15134-15141

25. Baynes JW (1991) The role of oxidative stress in diabetes. Diabetes 40: 405-412

26. Wolff SP, Jiang ZY, Hunt JV (1991) Protein glycation and oxidative stress in diabetes mellitus and ageing. Free Rad Biol Med 10: 339-352

27. Gallou G, Ruelland A, Legras B, Maugendre D, Allannic H, Cloarec L (1993) Plasma MDA in type 1 and type 2 diabetes. Clin Chim Acta 214: 227-234

28. Wierusz-Wysocka B, Wysocki H, Byks H, Zozulinska D, Wicretowicz A, Kazmierczak M (1995) Metabolic control quality and free radical activity in diabetic patients. Diabetes Res Clin Pract 27 (3): 193-197

29. Nourooz Zadeh J, Tajaddini Sarmadi J, McCarthy S, Betteridge DJ, Wolff SP (1995) Elevated levels of authentic plasma hydroperoxides in NIDDM. Diabetes 44 (9): 10541058

30. Paul RG, Bailey AJ (1996) Glycation of collagen: the basis of its central role in the late complications of aging and diabetes. Int J Biochem Cell Biol 28 (12): 1297-1310

31. Uchida K, Toyokuni S, Nishikawa K et al. (1994) Michael addition-type 4-hydroxy-2-nonenal adducts in modified low-density lipoproteins: markers for atherosclerosis. Biochemistry 33: 12487-12494

32. Davies KJA, Lin SW, Pacifici RE (1987) Protein degradation by oxygen radicals. IV. Degradation of denatured protein. J Biol Chem 262 (20): 9914-9920

33. Dean RT, Thomas SM, Vince G, Wolff SP (1986) Oxidation induced proteolysis and its possible restriction by some secondary protein modifications. Biomed Biochim Acta 45 (11-12): 1563-1573

34. Palinski W, Horrko S, Miller E et al. (1996) Cloning of monoclonal autoantibodies to epitopes of oxidized lipoproteins from apolipoprotein E-deficient mice. Demonstration of epitopes of oxidized low density lipoprotein in human plasma. J Clin Invest 98 (3): 800-814

35. Wells-Knecht KJ, Brinkmann E, Wells-Knecht MC et al. (1996) New biomarkers of Maillard reaction damage to proteins. Nephrol Dial Transplant 11 [Suppl 5]: 41-47

36. Kikugawa K, Beppu M (1987) Involvement of lipid oxidation products in the formation of fluorescent and crosslinked proteins. Chem Phys Lipids 44: 277-296

37. Esterbauer H, Jürgens G, Quehenberger O, Koller E (1987) Autoxidation of human low density lipoprotein: loss of polyunsaturated fatty acids and vitamin $\mathrm{E}$ and generation of aldehydes. J Lipid Res 28: 495-509

38. Singh S, Suri R, Agrawal CG (1995) Fluorescence properties of oxidised plasma low-density lipoproteins. Biochim Biophys Acta 1254: 135-139 